\title{
EXISTENCE OF DECAYING ENTIRE SOLUTIONS OF A CLASS OF SEMILINEAR ELLIPTIC EQUATIONS
}

\author{
TAKAŜI KUSANO ${ }^{1}$, EZZAT S. NOUSSAIR AND CHARLES A. SWANSON ${ }^{2}$
}

(Communicated by Walter Littman)

\begin{abstract}
The main result establishes the existence of a nontrivial nonnegative radial solution $u \in C_{\text {loc }}^{2}\left(\mathbf{R}^{N}\right)$ of a semilinear elliptic eigenvalue problem in $\mathbf{R}^{N}, N \geq 3$, such that $u(|x|)$ has uniform limit zero as $|x| \rightarrow \infty$. Asymptotic decay estimates and necessary conditions are obtained. Since such solutions do not exist in the space $W_{0}^{1,2}\left(\mathbf{R}^{N}\right)$, a considerable departure from standard procedures is required.
\end{abstract}

The existence of a number $\lambda>0$ and an associated nontrivial solution $u(x) \geq 0$ in $\mathbf{R}^{N}, N \geq 3$, will be proved for semilinear elliptic eigenvalue problems of the type $^{12}$

$$
\begin{aligned}
-\Delta u+p_{1}(|x|) f_{1}(u)= & \lambda p_{2}(|x|) f_{2}(u), \quad x \in \mathbf{R}^{N}, N \geq 3, \\
& \lim _{|x| \rightarrow \infty} u(x)=0,
\end{aligned}
$$

where $\Delta$ denotes the $N$-dimensional Laplacian and $p_{1}, p_{2}, f_{1}, f_{2}$ are locally Hölder continuous functions in $[0, \infty)$ satisfying growth conditions to be listed later. Such a solution $u \in C_{\mathrm{loc}}^{2}\left(\mathbf{R}^{N}\right)$ is termed a decaying entire solution of equation (1). The prototype with $f_{1}(u)=u^{\beta}, f_{2}(u)=u^{\gamma}, 1<\gamma<\beta$, and constants $p_{1}$, $p_{2}$ (i.e., $a=0$ in $\left(\mathrm{H}_{1}\right)$ below) has many well-known applications in quantum field theory, fluid mechanics, geometry, and other areas $[4,10,14,17]$.

For some $\lambda>0$, a nontrivial nonnegative entire solution $u(|x|)$ of (1) will be established satisfying the decay law

$$
u(|x|)=O\left(|x|^{(2-N) / 2}\right) \quad \text { as }|x| \rightarrow \infty .
$$

For the equation considered by Strauss [14]:

$$
-\Delta u+a_{0} u+f_{1}(u)=\lambda f_{2}(u), \quad a_{0}>0,
$$

and for more general equations $[2,12]$, the presence of a positive constant $a_{0}$ permits a variational approach to be used to construct a solution $u(|x|)$ in the Sobolev space $W_{0}^{1,2}\left(\mathbf{R}^{N}\right)$ which decays exponentially as $|x| \rightarrow \infty$. This of course cannot be true if $a_{0}=0$, the case under study here, as shown by easy examples. Generalizations and modifications of (4) considered by Berestycki and Lions [1, 2],

Received by the editors February 19, 1988.

1980 Mathematics Subject Classification (1985 Revision). Primary 35J60; Secondary 35B40.

Key words and phrases. Semilinear elliptic equation, bounded nonlinearity, entire nonnegative solution, asymptotic decay law.

${ }^{1}$ Supported in part by Grant-in-Aid for scientific Research (No. 61540114), Ministry of Education (Japan).

${ }^{2}$ Supported by NSERC (Canada). 
Berestycki, Lions, and Peletier [3], Berger [4], Ding and Ni [5], and others cited in these works, have character differing from (1) for the same reason.

The case $p_{1}(r) \equiv 0$ in (1), for which $\lambda$ is superfluous and can be deleted, has been widely studied, in particular by Gidas and Spruck [6], Kawano [7], Kusano and Naito [8], Kusano and Oharu [9], Ni [10], and Toland [15, 16]. Neither the results nor the methods used in this case appear to be cogent to the present problem.

The method to be used here proceeds as follows: (i) A sequence of differential equations (5) below is considered for which radial solutions $u_{k}$ in the space $W_{0}^{1,2}\left(\mathbf{R}^{N}\right)$ are obtained by the procedure of Strauss $[14], k=1,2, \ldots$.

(ii) Because of the direct variational method of constructing sequences $\left\{u_{k n}: n=\right.$ $1,2, \ldots\}$ which converge weakly in $W_{0}^{1,2}\left(\mathbf{R}^{N}\right)$ to $u_{k}$, a uniform bound on the $L^{2}\left(\mathbf{R}^{N}\right)$ norm of $\left|\nabla u_{k}\right|$ can be established.

(iii) Use of a uniform bound on $\left\{u_{k}\right\}$ establishes a subsequence of $\left\{u_{k}\right\}$ which converges in $C_{\text {loc }}^{2}\left(\mathbf{R}^{N}\right)$ to a nonnegative solution of (1) satisfying the decay law (3). The hypotheses for (1) are listed below, where

$$
g_{i}(s)=\int_{0}^{s} f_{i}(t) d t, \quad s \geq 0, i=1,2 .
$$

$\left(\mathrm{H}_{1}\right) p_{1}$ and $p_{2}$ are positive, bounded, locally Hölder continuous functions such that $p_{2}(r) \leq C_{0} p_{1}(r)$ and $p_{2}(r) \leq C /\left(1+r^{a}\right)$ in $0 \leq r<\infty$ for some constants $a \geq 0, C_{0}>0$, and $C>0$.

$\left(\mathrm{H}_{2}\right) f_{1}$ and $f_{2}$ are locally Hölder continuous in $[0, \infty), f_{1}(s) \geq 0$, and $f_{2}(s)>0$ for all $s>0$.

$\left(\mathrm{H}_{3}\right) f_{1}(s)=O(s), f_{2}(s)=O\left(s^{\gamma}\right)$ as $s \rightarrow 0+$, where $\gamma>1$ and

$$
\gamma>(N+2-2 a) /(N-2) \text { if } 0 \leq a \leq 2 .
$$

$\left(\mathrm{H}_{4}\right) f_{2}(s)=o\left(f_{1}(s)\right)$ and $f_{2}(s)=O\left(g_{1}(s) / s\right)$ as $s \rightarrow \infty$.

$\left(\mathrm{H}_{5}\right)$ There exist positive constants $C_{1}$ and $C_{2}$ such that $s f_{1}(s) \leq C_{1} g_{1}(s)$ and $s f_{2}(s) \geq C_{2} g_{2}(s)$ for all $s \geq 0$.

REMARK. The case $a>2$ also can be handled by a different procedure based on the existence of decaying entire positive solutions of linear elliptic equations and a comparison technique [13, Theorem 6]. This is possible in this case because $\int^{\infty} r p_{2}(r) d r<\infty$ by $\left(\mathrm{H}_{1}\right)$. We are unaware of any earlier methods which are applicable to the case $0<a \leq 2$.

THEOREM. Under these assumptions, there exists $\lambda>0$ and a corresponding nontrivial solution $u(|x|) \geq 0$ of (1) in $\mathbf{R}^{N}$ satisfying the decay law (3).

PROOF. Let $E$ denote the subspace of all radial functions in $W_{0}^{1,2}\left(\mathbf{R}^{N}\right)$. Consider the sequence of problems

$$
\begin{aligned}
& -\Delta u_{k}+(1 / k) u_{k}+p_{1}(r) f_{1}\left(u_{k}\right)=\lambda_{k} p_{2}(r) f_{2}\left(u_{k}\right), \\
& \qquad u_{k} \in E, r=|x| \geq 0, k=1,2, \ldots .
\end{aligned}
$$

Let $\left\{f_{i n}\right\}$ be the sequence of truncated functions defined by

$$
f_{i n}(s)= \begin{cases}f_{i}(s) & \text { if } s \leq n \\ f_{i}(n) & \text { if } s>n\end{cases}
$$


for $n=1,2, \ldots, i=1,2$, and define

$$
g_{\text {in }}(s)=\int_{0}^{s} f_{\text {in }}(t) d t, \quad s \geq 0, i=1,2 .
$$

A slight modification of Lemma 3 of Strauss [14, p. 157] shows, for fixed $k$ and $n$, that there exists a radial function $u_{k n}$ which minimizes the functional

$$
I_{k n}(u)=\frac{1}{2} \int_{\mathbf{R}^{N}}\left[|\nabla u|^{2}+\frac{u^{2}}{k}+2 p_{1} g_{1 n}(u)\right] d x
$$

subject to the constraints $u \in E$ and

$$
J_{n}(u)=\int_{\mathbf{R}^{N}} p_{2} g_{2 n}(u) d x=1 .
$$

By the Euler-Lagrange principle, there exists $\lambda_{k n} \in \mathbf{R}$ such that $I_{k n}^{\prime}\left(u_{k n}\right)=$ $\lambda_{k n} J_{n}^{\prime}\left(u_{k n}\right)$ in the dual space $E^{*}$, where the Fréchet derivatives are given by

$$
\begin{aligned}
I_{k n}^{\prime}(u) v & =\int_{\mathbf{R}^{N}}\left[\nabla u \cdot \nabla v+\frac{u v}{k}+p_{1} f_{1 n}(u) v\right] d x, \\
J_{n}^{\prime}(u) v & =\int_{\mathbf{R}^{N}} p_{2} f_{2 n}(u) v d x, \quad u, v \in E .
\end{aligned}
$$

(Clearly, $\lambda_{k n} \geq 0$ since the case $v=u$ is included.) This means that $u_{k n}$ is a weak solution of the problem

(8) $-\Delta u_{k n}+\frac{1}{k} u_{k n}+p_{1} f_{1 n}\left(u_{k n}\right)=\lambda_{k n} p_{2} f_{2 n}\left(u_{k n}\right)$

$$
u_{k n} \in E, k, n=1,2, \ldots
$$

A standard bootstrap argument implies in view of the regularity hypotheses $\left(\mathrm{H}_{1}\right)$, $\left(\mathrm{H}_{2}\right)$ that $u_{k n} \in C_{\mathrm{loc}}^{2+\alpha}\left(\mathbf{R}^{N}\right)$ for some $\alpha \in(0,1)$.

In analogy with (6), (7), the functionals associated with (5) are

$$
\begin{aligned}
I_{k}(u) & =\frac{1}{2} \int_{\mathbf{R}^{N}}\left[|\nabla u|^{2}+\frac{u^{2}}{k}+2 p_{1} g_{1}(u)\right] d x, \\
J(u) & =\int_{\mathbf{R}^{N}} p_{2} g_{2}(u) d x, \quad u \in E .
\end{aligned}
$$

Since $g_{2}(s)>0$ for all $s>0$ by $\left(\mathrm{H}_{2}\right)$, there exists a radial function $\phi \in C_{0}^{\infty}\left(\mathbf{R}^{N}\right)$ such that $J(\phi)=1$. If $n \geq \sup |\phi(x)|$, then $g_{i n}(\phi)=g_{i}(\phi), i=1,2$, and it follows from (6), (9), and the variational definition of $u_{k n}$ that

$$
I_{k n}\left(u_{k n}\right) \leq I_{1 n}(\phi)=I_{1}(\phi)<\infty .
$$

This implies that there exists a constant $M$, independent of $k$ and $n$, such that

$$
\int_{\mathbf{R}^{N}}\left|\nabla u_{k n}\right|^{2} d x \leq M
$$

and

$$
\left\|u_{k n}\right\|_{1,2}^{2} \leq 2 k M, \quad k, n=1,2, \ldots,
$$

where \|\|$_{1,2}$ denotes the norm in $E$. For fixed $k$, we conclude from (13) that $\left\{u_{k n}\right\}$ has a subsequence which converges weakly in $E$ to a limit $u_{k}$. The proof given by 
Strauss [14, pp. 159-160] shows that $u_{k}$ is a nontrivial nonnegative exponentially decaying entire solution of (5), where $\lambda_{k}$ in (5) is the limit of some subsequence of the (bounded) sequence $\left\{\lambda_{k n}: n=1,2, \ldots\right\}$. Closely related results appear in $[1,12]$. The weak convergence of $u_{k n}$ to $u_{k}$ in $L^{2}\left(\mathbf{R}^{N}\right)$ implies that $\partial u_{k n} / \partial x_{i}$ converges weakly in $L^{2}\left(\mathbf{R}^{N}\right)$ to $\partial u_{k} / \partial x_{i}$ for each $i=1, \ldots, N$, and consequently

$$
\int_{\mathbf{R}^{N}}\left|\nabla u_{k}\right|^{2} d x \leq \liminf _{n \rightarrow \infty} \int_{\mathbf{R}^{N}}\left|\nabla u_{k n}\right|^{2} d x .
$$

It follows from (12) and (14) that the sequence of norms $\left\|\nabla u_{k}\right\|_{L^{2}\left(\mathbf{R}^{N}\right)}$ is uniformly bounded. We now apply an estimate of Berestycki and Lions [2, Lemma AIII] for radial functions in $W_{0}^{1,2}\left(\mathbf{R}^{N}\right)$ :

$$
u_{k}(r) \leq A r^{(2-N) / 2}\left\|\nabla u_{k}\right\|_{L^{2}\left(\mathbf{R}^{N}\right)}, \quad r \geq 1,
$$

to conclude that there exists a constant $A^{\prime}$, independent of $k$, such that

$$
0 \leq u_{k}(r) \leq A^{\prime} r^{(2-N) / 2}, \quad r \geq 1 .
$$

To prove that $\left\{\lambda_{k}\right\}$ is bounded, we multiply (5) by $u_{k}$ and integrate by parts to obtain

$$
\int_{\mathbf{R}^{N}}\left[\left|\nabla u_{k}\right|^{2}+\frac{1}{k} u_{k}^{2}+p_{1} u_{k} f_{1}\left(u_{k}\right)\right] d x=\lambda_{k} \int_{\mathbf{R}^{N}} p_{2} u_{k} f_{2}\left(u_{k}\right) d x .
$$

In the limit $n \rightarrow \infty$, the constraint (7) on $u_{k n}$ becomes $J\left(u_{k}\right)=1$ (see (10)), and hence $\left(\mathrm{H}_{5}\right)$ shows that

$$
\int_{\mathbf{R}^{N}} p_{2} u_{k} f_{2}\left(u_{k}\right) d x \geq C_{2} \int_{\mathbf{R}^{N}} p_{2} g_{2}\left(u_{k}\right) d x=C_{2} J\left(u_{k}\right)=C_{2}>0 .
$$

Let $\tilde{C}_{1}=\max \left(C_{1}, 2\right)$, where $C_{1}$ is as in $\left(\mathrm{H}_{5}\right)$. Then the left side of $(16)$ is bounded above by

$$
\frac{1}{2} \tilde{C}_{1} \int_{\mathbf{R}^{N}}\left[\left|\nabla u_{k}\right|^{2}+\frac{1}{k} u_{k}^{2}+2 p_{1} g_{1}\left(u_{k}\right)\right] d x=\tilde{C}_{1} I_{k}\left(u_{k}\right)=\tilde{C}_{1} I_{1}(\phi),
$$

where $\phi$ is as in (11), and therefore (16) shows that $\left\{\lambda_{k}\right\}$ is bounded.

In view of the uniform estimate (15), there exists a subsequence of $\left\{u_{k}(r)\right\}$ which converges locally uniformly in $C^{2}\left(\mathbf{R}^{N}\right)$ to a function $u \in C_{\text {loc }}^{2}\left(\mathbf{R}^{N}\right)$. This is proved in the usual way from interior $L^{p}$-estimates and Schauder estimates on bounded domains in $\mathbf{R}^{N}$, permitting the selection of a convergent diagonal subsequence in $C_{\text {loc }}^{2}\left(\mathbf{R}^{N}\right)$. We choose a subsequence $\lambda_{k} \rightarrow \lambda \geq 0$ in (5) and conclude in the limit $k \rightarrow \infty$ that $u=\lim _{k \rightarrow \infty} u_{k}$ is a nonnegative entire solution of (1).

To prove that $u$ is nontrivial, first note from $\left(\mathrm{H}_{3}\right)$ and $\left(\mathrm{H}_{5}\right)$ that there exists a constant $C_{3}>0$ such that $0 \leq g_{2}(s) \leq C_{3} s^{\gamma+1}$ for $0 \leq s \leq 1$. Let $h(r)=r^{(2-N) / 2}$, so $h(r) \leq 1$ for $r \geq 1$. Then by $\left(\mathrm{H}_{1}\right)$ there is a constant $C_{4}>0$ such that

$$
p_{2}(r) g_{2}(h(r)) r^{N-1} \leq C_{4} r^{\delta} \quad \text { for } r \geq 1,
$$

where

$$
\delta=N-a-1+\left(\frac{2-N}{2}\right)(\gamma+1)=-\frac{(N-2) \gamma}{2}+\frac{N}{2}-a .
$$


It follows from the hypothesis on $\gamma$ in $\left(\mathrm{H}_{3}\right)$ that $\delta<-1$, and therefore $J(h)$ converges, i.e., by (10),

$$
J(h)=\int_{\mathbf{R}^{N}} p_{2}(|x|) g_{2}(h(|x|)) d x<\infty .
$$

Since $J\left(u_{k}\right)=1$ and $u_{k}$ satisfies (15), the dominated convergence theorem shows that

$$
J(u)=\lim _{k \rightarrow \infty} J\left(u_{k}\right)=1,
$$

and hence $u$ is not identically zero.

If $\lambda=0,(1)$ would imply that $u$ is a nontrivial nonnegative decaying entire solution of $-\Delta u \leq 0$, contradicting the maximum principle. Since obviously $\lambda \geq 0$, it follows that $\lambda>0$. The decay law (3) is immediate from (15). This completes the proof.

A lower bound for the solution $u(|x|)$ in the theorem is obtained under the additional conditions:

$\left(\mathrm{H}_{6}\right) p_{2}(r)=B p_{1}(r)$ for some constant $B>0, r \geq 0$;

$\left(\mathrm{H}_{7}\right) f_{1}(s)=o\left(f_{2}(s)\right)$ as $s \rightarrow 0+$.

COROLLARY 1. If $\left(\mathrm{H}_{1}\right)-\left(\mathrm{H}_{7}\right)$ hold, the solution $u$ in the theorem satisfies

$$
K_{1}|x|^{2-N} \leq u(|x|) \leq K_{2}|x|^{(2-N) / 2}, \quad|x| \geq 1,
$$

for some positive constants $K_{1}$ and $K_{2}$.

PROOF. It remains to prove the left inequality in (17). Let $r=|x|$. Since $u(r)$ satisfies (1) for some $\lambda>0$, for arbitrary $\varepsilon>0,\left(\mathrm{H}_{6}\right)$ and $\left(\mathrm{H}_{7}\right)$ show that there exists a corresponding $\delta>0$ such that

$$
-(\Delta u)(r)>(\lambda B-\varepsilon) p_{1}(r) f_{2}(u(r))
$$

whenever $u(r)<\delta$. Choose $0<\varepsilon<\lambda B$ and $R$ large enough that $u(r)<\delta$ for all $r>R$, possible by (3). Then $-(\Delta u)(r)>0$ for $r>R$. Since $0 \not z u(r) \geq 0$, the strong maximum principle implies that $u(r)>0$ for $r>R$. The left inequality (17) follows as a well-known consequence of the maximum principle.

The upper estimate in (17) can be sharpened i.e., (3) can be improved under the hypotheses $\left(\mathrm{H}_{1}\right)-\left(\mathrm{H}_{5}\right)$.

COROLlARY 2. The solution $u(|x|)$ in the theorem satisfies $u(r)=O\left(r^{-b}\right)$ as $r \rightarrow \infty$ for any constant $b$ in the interval

$$
\frac{N-2}{2}<b<\max \left\{\frac{(N-2) \gamma+2 a-4}{2}, N-2\right\} \text {. }
$$

ProOF. Since $u$ satisfies (1), $\left(\mathrm{H}_{1}\right),\left(\mathrm{H}_{3}\right)$, and (3) imply that there exists a constant $K>0$ such that

$$
-\Delta u \leq K r^{-\delta}, \quad r=|x| \geq 1
$$

where

$$
\delta=a+\frac{(N-2) \gamma}{2}=\frac{(N-2) \gamma+2 a}{2} .
$$


Consider the function $v_{A}(r)=A\left(1+r^{2}\right)^{-b / 2}$ for $b$ satisfying (18) and a constant $A$ to be chosen later. Define $D=\frac{1}{2} \min \{b N, b(N-b-2)\} ; D>0$, by (18). Differentiation yields

$$
-\Delta v_{A}=\frac{A b\left[N+(N-b-2) r^{2}\right]}{\left(1+r^{2}\right)^{(b+4) / 2}} \geq \frac{2 A D}{\left(1+r^{2}\right)^{(b+2) / 2}} .
$$

The choice of $b$ in (18) means that $b+2<\delta$. Then (19) and (20) imply the existence of a number $R \geq 1$ such that

$$
-\Delta v_{A} \geq \frac{A D}{r^{b+2}} \geq \frac{K}{r^{\delta}} \geq-\Delta u, \quad r \geq R .
$$

Now choose $A$ large enough that $v_{A}(R) \geq u(R)$. For such an $A$, it follows that

$$
\begin{aligned}
-\Delta\left(v_{A}-u\right) \geq 0 & \text { for }|x| \geq R, \\
v_{A}-u \geq 0 & \text { on }|x|=R, \\
v_{A}-u \rightarrow 0 & \text { as }|x| \rightarrow \infty,
\end{aligned}
$$

and the maximum principle implies that $v_{A}-u \geq 0$ throughout $\left\{x \in \mathbf{R}^{N}:|x| \geq R\right\}$. This means that $u(r) \leq C r^{-b}$ for some constant $C>0, r \geq R$.

NECESSARY CONDITIONS. The sharpness of our results can be tested from the known necessary condition $[\mathbf{1 1}, \mathrm{pp}$. 76-77]

$$
\int^{\infty} p(r) r^{N-1-\gamma(N-2)} d r<\infty
$$

for the existence of a positive solution $u(x)$ of the differential inequality $\Delta u+$ $p(r) u^{\gamma} \leq 0$ in an exterior domain $\Omega_{R}=\left\{x \in \mathbf{R}^{N}:|x|=r>R\right\}, N \geq 3$. As in the proof of Corollary 1, if $\left(\mathrm{H}_{6}\right)$ and $\left(\mathrm{H}_{7}\right)$ hold, a nontrivial nonnegative solution $u$ of (1), (2) satisfies

$$
-(\Delta u)(x) \geq \lambda_{1} p_{2}(r) f_{2}(u(x))
$$

for all sufficiently large $r=|x|$, say $r>R$, for some positive constant $\lambda_{1}$. Since $-\Delta u \geq 0$ in $\Omega_{R}$ by (22), the strong maximum principle shows that $u>0$ in $\Omega_{R}$. If there exist positive constants $C$ and $K$ such that

$$
p_{2}(r) f_{2}(s) \geq C r^{-a} s^{\gamma}, \quad r>R, 0<s \leq K,
$$

then (21) implies the necessary condition $-a+N-1-\gamma(N-2)<-1$, equivalent to

$$
\gamma>\frac{N-a}{N-2}, \quad N \geq 3 .
$$

EXAMPLE. Application of the existence theorem and the necessary condition (23) to the prototype

$$
\begin{gathered}
-\Delta u=\frac{\lambda u^{\gamma}-u^{\beta}}{1+r^{a}}, \quad \gamma<\beta, a \geq 0, \\
\lim _{|x| \rightarrow \infty} u(x)=0
\end{gathered}
$$

yields the following conclusions:

(i) If $a=2$, the condition $\gamma>1$ is necessary and sufficient for the existence of a nontrivial nonnegative solution of (24) in $\mathbf{R}^{N}$ for some $\lambda>0$. 
(ii) If $a>2$, the condition $\gamma>1$ is sufficient for the existence of such a solution.

(iii) If $0 \leq a<2$, no such solution exists if $\gamma \leq(N-a) /(N-2)$, but it does exist if $\gamma>(N+2-2 a) /(N-2)$.

The case

$$
\frac{N-a}{N-2}<\gamma \leq \frac{N+2-2 a}{N-2}
$$

remains open. Our results can be extended to generalizations of (1) of the type

$$
-\Delta u+f_{1}(|x|, u)=\lambda f_{2}(|x|, u), \quad x \in \mathbf{R}^{N}
$$

under suitable technical hypotheses on $f_{1}$ and $f_{2}$, by the same procedure. Also, $\Delta$ could be replaced by a selfadjoint linear uniformly elliptic operator of second order. The method also applies to boundary value problems for (1) or (25) in exterior domains $\Omega_{R}$ in $\mathbf{R}^{N}$.

\section{REFERENCES}

1. H. Berestycki and P. L. Lions, Une méthode locale pour l'existence de solutions positives de problèmes semi-linéaires elliptiques dans $\mathbf{R}^{N}$, J. Analyse Math. 38 (1980), 144-187.

2. $\_$, Nonlinear scalar field equations. I, Arch. Rational Mech. Anal. 82 (1983), 313-345.

3. H. Berestycki, P. L. Lions, and L. A. Peletier, An ODE approach to the existence of positive solutions for semilinear problems in $\mathbf{R}^{N}$, Indiana Univ. Math. J. 30 (1981), 141-157.

4. M. S. Berger, On the existence and structure of stationary states for a nonlinear Klein-Gordon equation, J. Funct. Anal. 9 (1972), 249-261.

5. W.-Y. Ding and W.-M. Ni, On the existence of positive entire solutions of a semilinear elliptic equation, Arch. Rational Mech. Anal. 91 (1986), 283-308.

6. B. Gidas and J. Spruck, Global and local behaviour of positive solutions of nonlinear elliptic equations, Comm. Pure Appl. Math. 34 (1981), 525-598.

7. N. Kawano, On bounded entire solutions of semilinear elliptic equations, Hiroshima Math. J. 14 (1984), 125-158.

8. T. Kusano and M. Naito, Positive entire solutions of superlinear elliptic equations, Hiroshima Math. J. 16 (1986), 361-366.

9. T. Kusano and S. Oharu, On entire solutions of second order semilinear elliptic equations, J. Math. Anal. Appl. 113 (1986), 123-135.

10. W.-M. Ni, On the elliptic equation $\Delta u+K(x) u^{(n+2) /(n-2)}=0$, its generalizations, and applications in geometry, Indiana Univ. Math. J. 31 (1982), 493-529.

11. E. S. Noussair and C. A. Swanson, Oscillation theory for semilinear Schrödinger equations and inequalities, Proc. Royal Soc. Edinburgh 75A (1975/76), 67-81.

12. _ Positive solutions of semilinear elliptic problems in unbounded domains, J. Differential Equations 57 (1985), 349-372.

13. __ Positive solutions of elliptic systems with bounded nonlinearities, Proc. Royal Soc. Edinburgh 108A (1988), 321-332.

14. W. A. Strauss, Existence of solitary waves in higher dimensions, Comm. Math. Phys. 55 (1977), 149-162.

15. J. F. Toland, On positive solutions of $-\Delta u=F(x, u)$, Math. Z. 182 (1983), 351-357.

16. __ Positive solutions of nonlinear elliptic equations-existence and nonexistence of solutions with radial symmetry in $L_{p}\left(\mathbf{R}^{N}\right)$, Trans. Amer. Math. Soc. 282 (1984), 335-354.

17. J. S. W. Wong, On the generalized Emden-Fowler equation, SIAM Rev. 17 (1975), 339-360.

Department of Mathematics, Faculty of Science, hiroshima University, HIROSHIMA 730, JAPAN

School of Mathematics, University of New South Wales, Kensington, NeW South Wales, Australia 2033

DEPARTMENT OF MATHEMATICS, UNIVERSity OF BRITISH COlUMBIA, VANCOUVER, BRITISH COLUMBia, CANADA V6T 1Y4 